Recent architecture,

competitions and public space. A landscape of lights and shadows

\section{RESUMEN}

Durante los últimos años hemos asistido a una persistente presencia de la arquitectura chilena contemporánea en distintos medios de difusión a nivel internacional. Habiendo alcanzado un nivel de madurez reconocido a través de diversos premios y distinciones, la producción de nuestros arquitectos revela una calidad destacada dentro del panorama latinoamericano.

Sin embargo, también es posible constatar que bajo este panorama de luces, el fenómeno antes descrito no alcanza a retratar de manera integra el estado de situación local, toda vez que la arquitectura de uso público ha quedado confinada a un lugar secundario, y lo que es más preocupante, las obras señaladas por la crítica como de calidad sobresaliente constituyen una excepción en el paisaje de nuestras ciudades, que aún cargan con un horizonte sombrío en relación a los estándares de calidad de vida urbana.

\section{ABSTRACT}

During the last few years we have witnessed a persistent presence of contemporary Chilean architecture in the international mass media. Having achieved a level of maturity, recognized through different awards and distinctions, the current production of Chilean architects reveals a high standard of quality, one of the highest in the Latin American scene.

However, it is also possible to appreciate that under this highlighted scene, the phenomenon previously described cannot be used to understand the whole scene of Chilean architecture.

\title{
Arquitectura reciente, concursos y espacios de uso público. Un paisaje de luces y sombras
}

\section{SOBRE LA PREEMINENCIA DEL ÁMBITO DE LO PRIVADO EN EL ESTADO DE LA ARQUITECTURA CHILENA RECIENTE}

Durante las últimas dos décadas, el estado de situación de la arquitectura chilena ha supuesto una firme presencia a nivel global. Publicaciones de obras de arquitectos nacionales en diversas revistas especializadas de gran prestigio, reseñas en medios de comunicación masivos, la participación en muestras de arquitectura internacionales y la representación en bienales, no han hecho sino acrecentar el prestigio de nuestra producción e hilvanar un panorama auspicioso sobre su calidad. La reciente adjudicación del León de Plata para el Pabellón de Chile en la versión número catorce de la Bienal de Arquitectura de Venecia, cuya curatoría y ejecución estuvo a cargo de Pedro Alonso y Hugo Palmarola; el encargo realizado a Smiljan Radic para el diseño del pabellón de la Galería Serpentine en Londres; o la selección por parte de la Real Academia de Artes de Londres de la oficina Pezo Von Ellrichshausen para participar en la exhibición Sensing Spaces, en enero de 2014, constituyen las muestras más recientes de la exitosa internacionalización de prominentes arquitectos chilenos.

La hipótesis sobre el prestigio y la calidad alcanzada por la arquitectura en nuestro país ha sido ampliamente tratada por varios autores, quienes postulan desde una emergencia generada luego de un período en que las relaciones y el intercambio cultural con el extranjero estuvieron estancados (Mardones, 2008: 42), hasta una explicación que define la clave del éxito a partir de una mirada menos interesada en los modelos foráneos, asumiendo "sus propias condiciones, su paisaje como referente y desechando la tecnología ultrasofisticada, para incluir materiales naturales, no por nostalgia, sino por un sentido práctico" (Adriá, 2011: 24).

Sin embargo, detrás de todo el esplendor que supone la difusión de la arquitectura reciente de nuestro país en el extranjero y el consiguiente reconocimiento de sus atributos, este fenómeno al parecer no alcanza a retratar de manera cabal el estado de situación local, toda vez que la arquitectura de uso público ha quedado

Rodrigo Aguilar (Viña del Mar, 1974) es Arquitecto de la Universidad de Chile (1998) y Máster en Historia, Arte, Arquitectura y Ciudad por la ETSAB | Universidad Politécnica de Cataluña (2001). Desde 1998 imparte clases en la Facultad de Arquitectura y Urbanismo de la Universidad de Chile y desde 2006 es Profesor en la Universidad de Santiago, donde actualmente se desempeña como Subdirector Académico de la Escuela de Arquitectura. Ha sido además Profesor Invitado en las Universidades Central y de Talca. Desde 2001 trabaja en su propio estudio en Santiago de Chile. En 2008 recibe el Premio Arquitecto Joven, otorgado por el Colegio de Arquitectos de Chile. 
confinada a un lugar secundario, y lo que es más preocupante, las obras señaladas por la crítica como sobresalientes constituyen una excepción en el paisaje de nuestras ciudades, que todavía cargan con muchos problemas relacionados con equidad urbana y acceso a espacios y edificios de uso público de calidad.

Esta situación de asimetría ya se ha manifestado de manera incipiente, señalándose a modo de ejemplo que "cualquiera que haya hojeado el último número de la prestigiosa publicación japonesa $a+u$ dedicado a Chile probablemente terminó con la impresión de que esta es básicamente una región de increíbles paisajes poblado por gemas arquitectónicas habitadas por un público de clase media-alta" (Palma,

2006: 32). De lo anterior se desprende que el ámbito de lo privado es el lugar preponderante donde se ha venido sistemáticamente expresando la calidad de nuestra arquitectura reciente.

En efecto, si realizamos un breve análisis cuantitativo de un libro canónico para la arquitectura chilena de los últimos años como "Blanca Montaña", nos daremos cuenta que el $81 \%$ de las obras presentadas corresponden a encargos privados, destacándose principalmente programas de segunda vivienda, hoteles en lugares de alta calidad paisajística y edificios corporativos, relegándose solo una parte menor de los trabajos exhibidos a iniciativas impulsadas por el Estado para la edificación pública.

La observación antes descrita deja ver entonces una paradoja: mientras la arquitectura chilena recibe premios internacionales y es reconocida universalmente, la huella dejada por una arquitectura de carácter más masivo en nuestras ciudades en términos generales sigue estando por debajo de los estándares de calidad. En esta línea argumental, parece pertinente preguntarse żcómo al cabo de las últimas décadas la arquitectura privada ha desplazado a la arquitectura pública en un rol trascendente?, y más específicamente żpor qué no ha existido una conciliación entre la calidad de la arquitectura y un mejoramiento del espacio urbano en nuestras ciudades?, żes posible encontrar uno o varios factores que expliquen el aún reducido impulso de la edificación de los espacios de uso público en el contexto actual?

Para dilucidar estos cuestionamientos, habría que poner atención en primer término a un contexto histórico general. No es difícil adivinar que la preeminencia de la arquitectura de carácter privado haya encontrado en nuestro país un campo fértil para su desarrollo. El modelo económico de corte neoliberal heredado desde mediados de la década de los ochenta, supuso un acento en la iniciativa privada como motor de expansión del desarrollo, relegando al Estado a un rol con carácter más bien regulador y con un peso cada vez más disminuido como impulsor de iniciativas que propendieran al mejoramiento de la ciudad y de la edificación pública.

Sin embargo, es importante señalar que en una perspectiva más específica, habría que indagar en el sistema general de acceso al diseño de proyectos de la esfera pública para encontrar algunas luces sobre la deuda de la arquitectura con la mejora en la producción de espacios para la ciudadanía.

\section{SOBRE EL SISTEMA DE ACCESO A LA ARQUITECTURA DE USO PÚBLICO}

El sistema de acceso a la edificación de uso público en nuestro país es complejo y ha sido producto de un enmarañado campo que se solidifica en los decretos del Ministerio de Obras Públicas y se decanta en el año 2003, con la promulgación de la Ley de Compras Públicas. Desde esta perspectiva, para el Estado los proyectos de diseño arquitectónico se definen como adquisiciones, tanto como objetos, bienes o servicios, que son adquiridos mediante contratos administrativos que se celebran previa ejecución de una licitación pública y que permiten elegir al oferente del servicio solicitado luego de la examinación de una serie de aspectos administrativos y técnicos.

De esta forma, la licitación pública se entiende como un procedimiento administrativo con el carácter de concurso, a partir del cual Ministerios, Municipalidades y otros órganos del Estado realizan un llamado público, pero a la vez restringido a la inscripción en registros de consultores y otros requisitos definidos con antelación, convocando a los interesados para que, sujetos a las bases fijadas, formulen propuestas entre las cuales se seleccionará y aceptará la más conveniente. El problema está en que esta elección de propuestas integra entre los factores de elegibilidad consideraciones de menor precio en la adquisición del servicio, aspectos de tiempo en la concreción del proyecto y variables enfocadas a examinar la idoneidad del currículo y trayectoria del oferente que lo capacita para emprender el diseño solicitado. A estos aspectos se puede agregar, sin que sea obligatorio legalmente, una variable que considere el diseño arquitectónico (que para efectos de lo solicitado puede ir desde una idea general contenida en un bosquejo hasta un anteproyecto) como un factor más dentro del la larga lista que suponen los criterios de evaluación. Por otra parte, el examen de las propuestas es realizado por una comisión técnica, que no considera dentro de su composición un jurado representante de los postulantes a la licitación, y que muchas veces tiende a privilegiar aspectos administrativos y procedimentales en la elaboración del proyecto, más que atender a los atributos propios del diseño arquitectónico presentado.

Una derivación del sistema antes descrito, amparado bajo la Ley de Concesiones, permite la contratación ya no solo del diseño sino de la ejecución de las obras de infraestructura del Estado, bajo la modalidad denominada Sistema de Pago Contra Recepción, que consiste en que el pago se realiza de acuerdo al recibo de las etapas de la obra que han sido acabadas, fiscalizadas y que tengan el visto bueno para ser entregadas. Esta modalidad resulta aún más árida como campo para el florecimiento de arquitecturas comprometidas con el mejoramiento sustancial de los espacios de uso público, puesto que la formulación del diseño constituye aquí solo un apéndice dentro del conjunto de antecedentes que entrega la empresa constructora o del consorcio que 


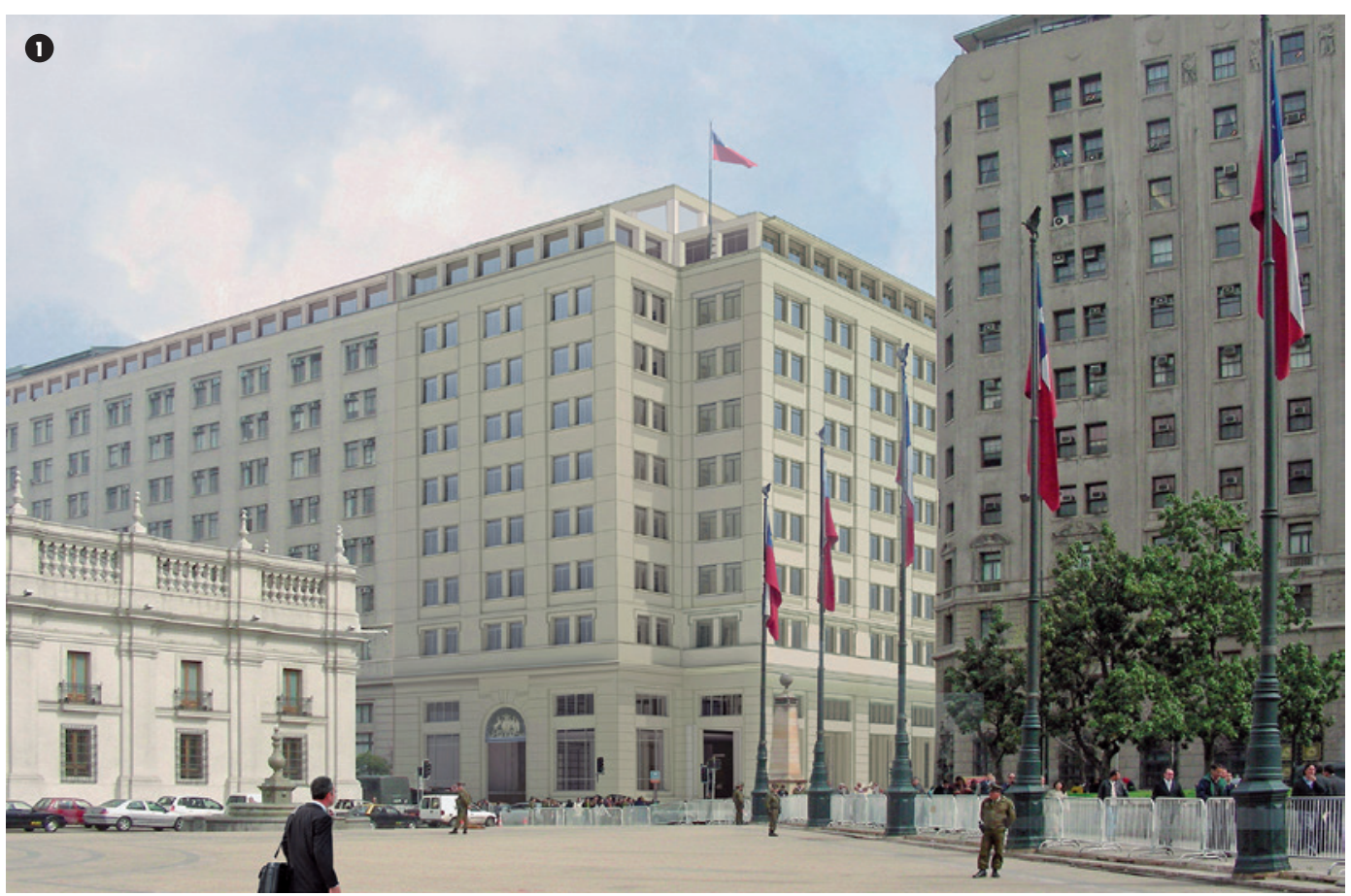

postula a la adjudicación del contrato, quien es además la responsable legal de su ejecución. Es importante destacar que esta modalidad nace originalmente para la construcción de grandes infraestructuras de ingeniería, como autopistas urbanas y que en los últimos años ha incorporado obras como cárceles y hospitales, en los cuales el proyecto de arquitectura siendo una pieza fundamental, ha quedado supeditado a las decisiones económicas y al manejo financiero de los respectivos contratos. Los persistentes atrasos en la ejecución de las obras y los aumentos presupuestarios, producto de que al momento de adjudicar los trabajos no se encuentran definidos a cabalidad los detalles y especialidades concurrentes al diseño de arquitectura, han puesto en duda además la operatividad del modelo.

Un ejemplo reciente de este hecho es el caso del Edificio Moneda Bicentenario, inmueble en ejecución que se levanta en la esquina norponiente del Palacio de La Moneda e impulsado como la primera obra del programa Legado Bicentenario. Con una superficie edificada de $39.000 \mathrm{~m}^{2}$ y destinado a dar cabida a una serie de dependencias ligadas al gobierno central, el proceso se inició en enero de 2011 , con la aprobación por parte del Ministerio de Obras Públicas de las bases de licitación, adjudicándose el proyecto en el mes de abril del mismo año, por un monto inferior al $39 \%$ del presupuesto calculado en las bases de licitación y reduciéndose el plazo original de 750 a 660 días para su recepción final. Sin embargo, una serie de retrasos en las aprobaciones de las etapas intermedias han aplazado el término de la obra que originalmente debería haber sido entregada en agosto de 2013

Ocurre entonces aquí un descalce entre lo que se espera para una arquitectura de uso público en términos cualitativos, y un sistema de acceso a la ejecución de dicha arquitectura que opera con criterios cuantitativos y que deja de lado el juicio a las consideraciones propias del diseño con incidencias directas en la construcción de un entorno adecuado y pertinente para la ciudadanía. Dicho de otra forma, asumiendo que "en el campo de la arquitectura es ampliamente aceptado que las propuestas de diseño arquitectónico sean evaluadas en razón del mérito y la calidad" (Molina, 2014: 21), los procedimientos de selección para estas propuestas en el ámbito de lo público en nuestro país distan de arrogarse esta consideración.

Una tercera vía de ejecución de arquitecturas de uso público, donde asume de forma más visible el factor de calidad del diseño, corresponde al establecimiento de concursos de ideas y anteproyectos de arquitectura abiertos, promocionados ya sea por gobiernos locales o por la administración central y patrocinados por el Colegio de Arquitectos. Sin embargo, estos concursos abiertos todavía ocurren en situaciones particulares, generalmente asociadas a la ejecución de obras emblemáticas que no logran completar el tejido urbano, sino que se visualizan desde la lógica de piezas excepcionales orientadas a la construcción de la imagen de la ciudad y su repercusión en ámbitos de competitividad global. Un segundo riesgo asociado es que estas instancias no siempre aseguran la construcción de la obra tal y como se presenta, pues no siempre está enlazado el resultado del concurso con la posterior contratación del desarrollo del proyecto.

\section{SOBRE LOS CONCURSOS COMO IMPULSORES DE LA CALIDAD EN LA ARQUITECTURA DE USO PÚBLICO}

No obstante lo expuesto anteriormente, es posible observar que al cabo de la última década, los concursos de arquitectura han comenzado a tener un dinamismo mayor en nuestro país y han alcanzado una preeminencia que de alguna manera comienza a superar un período considerable de tiempo en el cual esta modalidad constituyó más bien una singularidad. Una noticia auspiciosa al respecto es que desde hace algunos años, los Fondos para la Cultura y las Artes hayan considerado dentro de sus líneas de trabajo, la apertura de recursos para fomentar la promoción de concursos públicos de arquitectura y otros organismos del Estado, como el Ministerio de Obras Públicas, el Ministerio de la Vivienda y diversas Municipalidades, hayan impulsado de forma más sistemática la generación de estas 
2. Propuesta ganadora Concurso Plan Maestro Eje Bulnes. Fotomontaje. Cristián Undurraga. Fuente: www. plataformaurbana.c

iniciativas, amparados por el patrocinio del Colegio de Arquitectos de Chile y asumiendo su Reglamento de Concursos. Conviene además destacar en este apartado el estudio del sistema de licitaciones públicas para el fortalecimiento de los concursos de arquitectura en Chile, encargado el año 2012 por el Consejo Nacional de la Cultura y las Artes a Christián Matus, Maximiano Atria y Horacio Torrent, que busca identificar las fortalezas y debilidades además de generar una propuesta de mejoramiento para el perfeccionamiento en los procesos de concursabilidad para la arquitectura pública.

Estos antecedentes constituyen sin duda una noticia esperanzadora para la disciplina en el más amplio espectro de los programas de arquitectura pública a abordar, toda vez que en primera instancia los concursos abiertos permiten una mirada crítica sobre la especificidad del problema a resolver a nivel particular y -lo que es más importante-, suponen una reflexión profunda sobre los campos de preocupación de la arquitectura, posibilitando la asunción de respuestas innovadoras y con una impronta de calidad. Asumiendo en primera instancia que "el concurso vive en la cultura neoliberal su hora más difícil" (Montealegre B., 2007: 78), puesto que la selección de un proyecto por medio de la celebración de un evento de esta naturaleza depende de una cierta convergencia de fines, básicamente radicada entre los intereses del promotor del concurso y lo que podríamos definir como bien común; y tomando en cuenta algunos puntos de vista menos optimistas sobre estas instancias como oportunidades en las cuales se desplaza la opción de participación para transformarse en modalidades de contratación un tanto oportunistas y eminentemente especulativas (Matute P., 2010: 77), es necesario precisar que principalmente para la esfera de la arquitectura de uso público, los concursos abiertos siguen constituyendo una alternativa pertinente para explorar las mejores opciones posibles a aquello que es de interés colectivo.

En otro ámbito, los concursos de arquitectura corresponden a instancias donde es posible

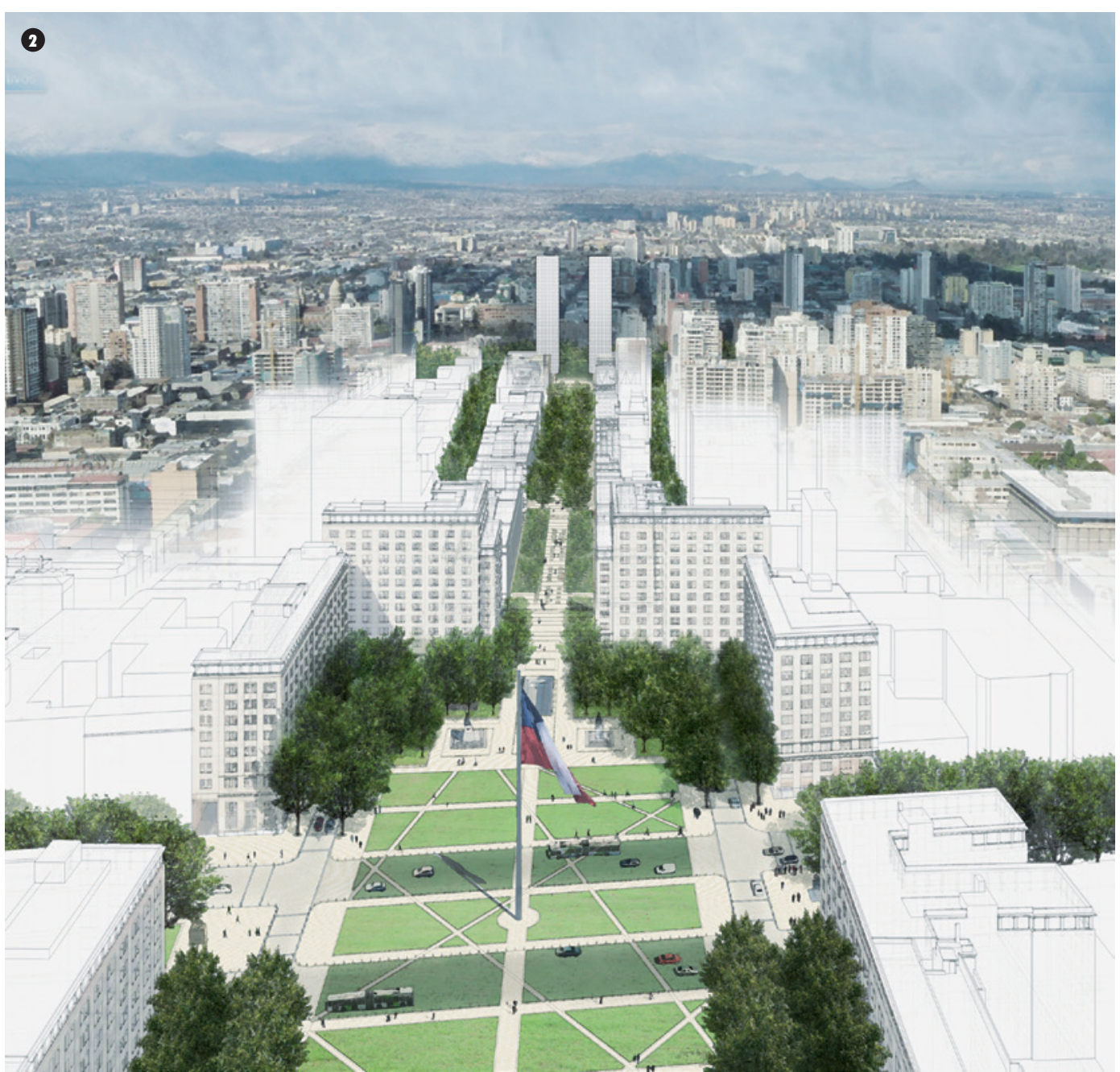

poner a disposición por parte de un gran contingente de capital humano, la resolución más idónea relativa al espacio habitable. En esta dirección, "la arquitectura corresponde a una disciplina en que el proyecto, como visión anticipada de la obra, tiene una importancia fundamental y donde la magnitud de los recursos implicados favorece el esfuerzo por asegurar con antelación la calidad del resultado" (Pérez, 2007: 10). El sistema de concursos entonces constituye a nuestro juicio la mejor forma que tiene la ciudadanía y un cuerpo de expertos y especialistas, para elegir qué y cómo quiere construir sus lugares más significativos. Esta consideración es de vital importancia, puesto que los concursos "dan cuenta de una suma de acciones arquitectónicas, urbanas y culturales, que laboriosamente permiten definir el carácter de la obra pública. Se trata de la búsqueda de un relativo consenso alrededor de los símbolos que nos evocan pertenencia e identidad en el territorio" (Piga, 2008: 11)

En la misma línea argumental, la promoción y adecuada regulación de concursos abiertos pueden entenderse también como el mecanismo más idóneo para la ejecución de obras de uso público que no solo puedan ser dignas de merecido reconocimiento, sino que además puedan tener como fin el mejoramiento sustancial del entorno urbano. En este sentido, 
3. Concurso Torre Antena Santiago. Smiljan Radic y equipo. Fuente: www.plataformaarquitectura.cl

4. Concurso Torre Antena Santiago. Smiljan Radic y equipo. Vista Nocturna. Fuente: www. plataformaarquitectura.cl conviene insistir en el incentivo de los concursos en el más amplio abanico de posibilidades y escalas, puesto que, tal como mencionábamos anteriormente, en los últimos años se ha observado que estos procedimientos se han llevado a cabo más bien para ejecutar obras de carácter emblemático y en algunos casos para la producción de íconos cuyos fines se acercan más al posicionamiento de la ciudad en rankings internacionales con un sesgo de orden publicitario, con la visión de constituir un centro de atracción que impulsa la posterior llegada de inversiones que apuestan por el desarrollo de mercados emergentes.

Como ejemplo de esta situación podemos mencionar el concurso internacional para la ejecución del Plan Maestro del Eje Bulnes, llevado a cabo en el año 2012 e impulsado desde el gobierno de Sebastián Piñera bajo el denominado Legado Bicentenario, instancia que propendía a la generación, revitalización y consolidación de espacios públicos y edificaciones de trascendencia urbana y patrimonial para el país. El concurso comprendió la intervención sobre el eje cívico al sur de la Alameda Bernardo O'Higgins y el Parque Almagro, entendido este último como remate de dicho eje. La propuesta ganadora, cuya autoría corresponde a la oficina de Cristián Undurraga incluyó la decisión de proponer un remate del paseo eje Bulnes a escala urbana, concebido como parte de la edificación del mismo y constituido por dos torres de 100 metros de altura, modificando la escala de los cuerpos edificados existentes.

En esta misma dirección, aunque con algunos matices, conviene citar el concurso de alcance internacional para la Torre Antena Santiago, convocado durante el año 2013, y que junto con la construcción de una estructura cuyo propósito consistía en localizar las numerosas estructuras de comunicación que actualmente se ubican sobre las cumbres del cerro San Cristóbal en un único edificio, también esperaba la constitución de una torre cuyo fin era convertirse en un hito urbano de la ciudad de Santiago y punto de atracción de visitantes. Afortunadamente, el proyecto

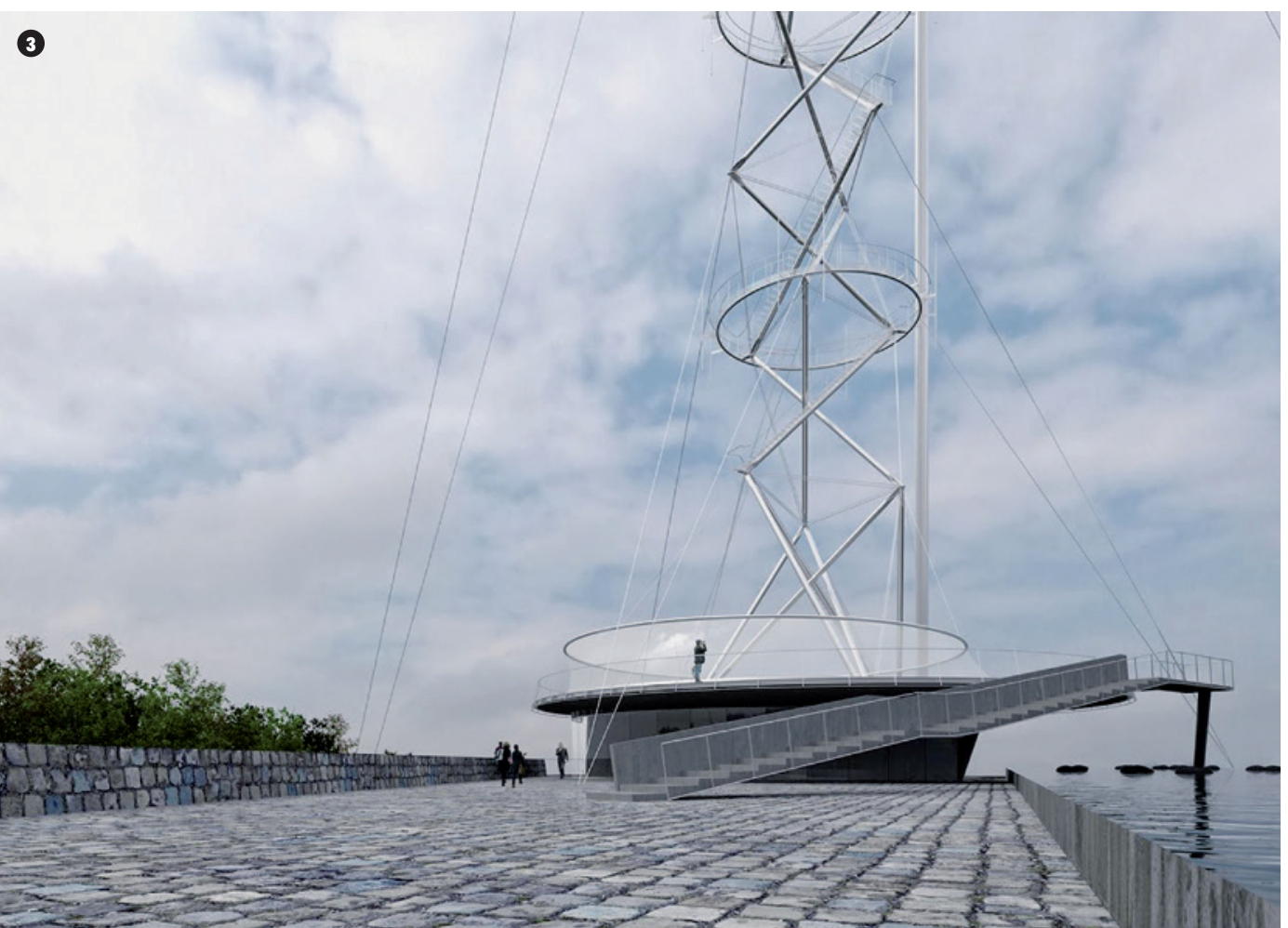

4
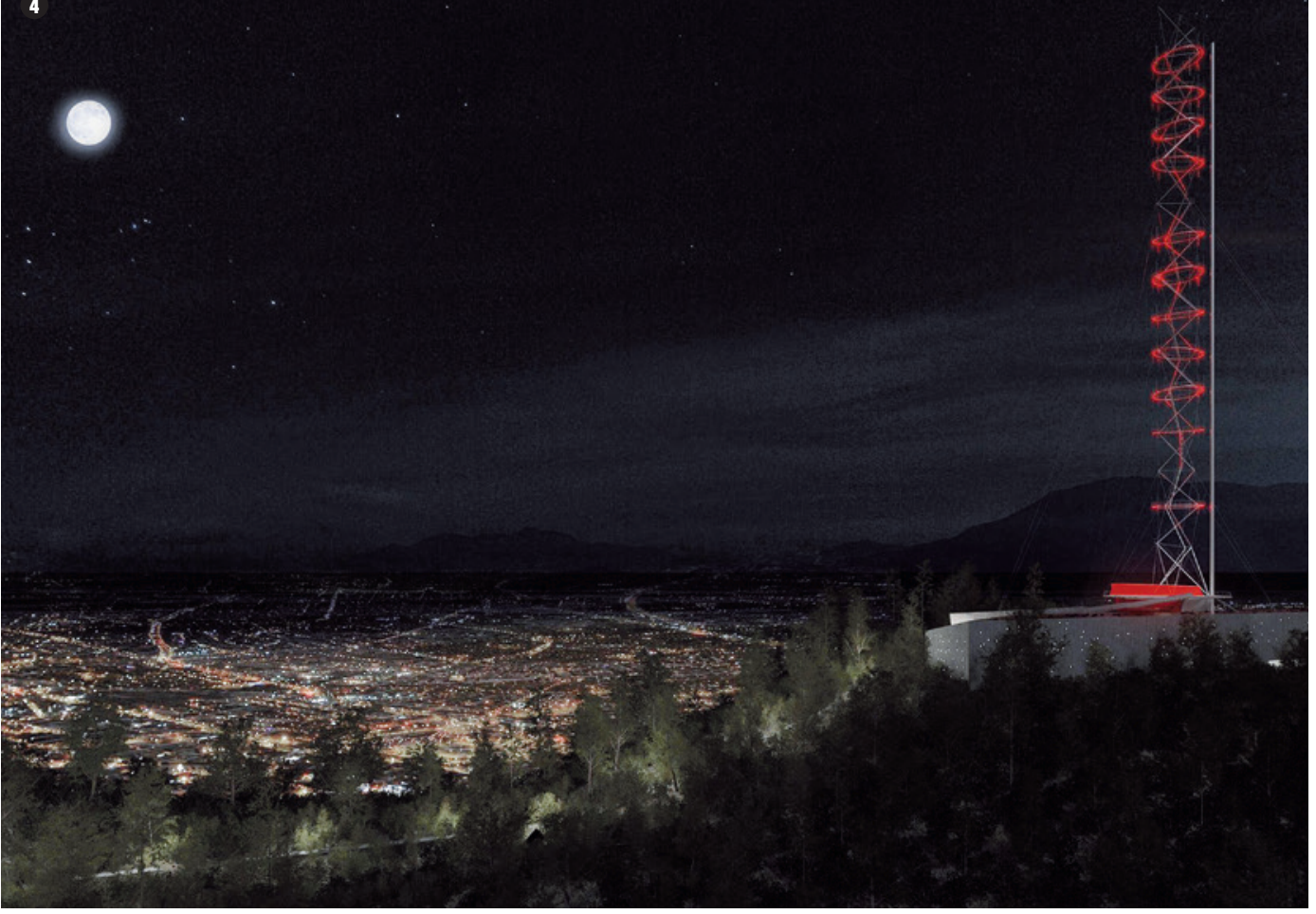
ganador cuya autoría corresponde al equipo encabezado por el arquitecto Smiljan Radic, logra sortear con delicadeza el cometido, mediante una pieza que a través de la lógica de elementos dispuestos en tracción, permite la configuración de una estructura etérea y leve sin menoscabar el monumento existente de remate del cerro y aprovechando de paso la privilegiada ubicación para configurar espacios de observación del entorno. Es necesario destacar que en estos casos no se trata se establecer un cuestionamiento sobre las respuestas arquitectónicas, que especialmente en el caso del último concurso mencionado, han sido tratadas con extremo rigor y que han significado un aporte innegable desde el punto de vista de sus cualidades, sino más bien es conveniente preguntarse sobre el espíritu de las bases elaboradas y su manifiesta intención de establecer obras de carácter icónico allí donde es discutible su emplazamiento.

Un caso contrario al relatado en los párrafos precedentes es la excepcional valoración del caso de Medellín en los últimos años, y el impacto positivo que han tenido los concursos de arquitectura en la regeneración de la ciudad y la calidad de vida de sus habitantes. Teniendo como base los lineamientos definidos en los denominados Proyectos Urbanos Integrales, que han tomado en consideración herramientas de desarrollo social, físico y la coordinación interinstitucional para transformar los sectores de la ciudad que poseían mayores necesidades. La mayor parte de las arquitecturas destacadas y difundidas por la crítica especializada en este caso, han sido fruto de concursos públicos, llevados a cabo gracias a una sinergia de las autoridades que han visualizado que es perfectamente posible cambiar el rostro a extensos sectores de la ciudad a través de una agresiva y sostenida política de mejoramiento urbano. Esta iniciativa, acompañada de proyectos detonantes que se han ejecutado vía concursos, han viabilizado un mejoramiento de las condiciones de equidad urbana, regenerando barrios completos que antes se encontraban en franco proceso de deterioro. No es de extrañar que varios de los arquitectos más destacados de Colombia en los últimos años hayan iniciado sus carreras con la ejecución de estos concursos.

\section{REFLEXIONES FINALES}

A la luz de los antecedentes antes examinados, podemos afirmar que nos encontramos ante un panorama de luces y sombras sobre el problema de la visibilidad, valoración y calidad de las obras de uso público en relación al prestigio y reconocimiento generalizado alcanzado por la arquitectura de nuestro país.

Por una parte, una no despreciable porción de proyectos públicos es accesible a través de un sistema de licitaciones que pone énfasis en aspectos cuantitativos y de oferta económica, lo que impide una apreciación de los aspectos cualitativos del diseño arquitectónico que se oferta. Esta situación es llevada a su máxima expresión mediante la modalidad de pago contra recepción, utilizado principalmente en obras concesionadas, donde el proyecto de arquitectura es solo un añadido, puesto que nuevamente lo que se impone es el análisis financiero de las propuestas. Por otra parte, los concursos abiertos y con resguardo del anonimato, que podrían ser una muy buena alternativa para la germinación de arquitectura de uso público con atributos de excelencia, aún teniendo un resurgimiento durante los últimos años, todavía constituyen casos excepcionales a la norma, siendo utilizados básicamente para obras de carácter monumental o bien para edificios y espacios públicos relevantes para la ciudad.

En consideración a lo anteriormente expuesto, resulta urgente revisar, reformular y perfeccionar el sistema de licitaciones y concursos desde las políticas públicas, incentivando y extendiendo esta última modalidad para obras y espacios de mediana y pequeña envergadura. En esta línea, cabe mencionar además que existe todavía un abultado monto de inversión pública que no ha sido debidamente aprovechado para convocar y desarrollar una arquitectura de uso público de calidad.
5. Licitación Edificio Fiscalía Nacional. Lateral Arquitectos + Luis Corvalán. Fuente: www.plataformaarquitectura.cl

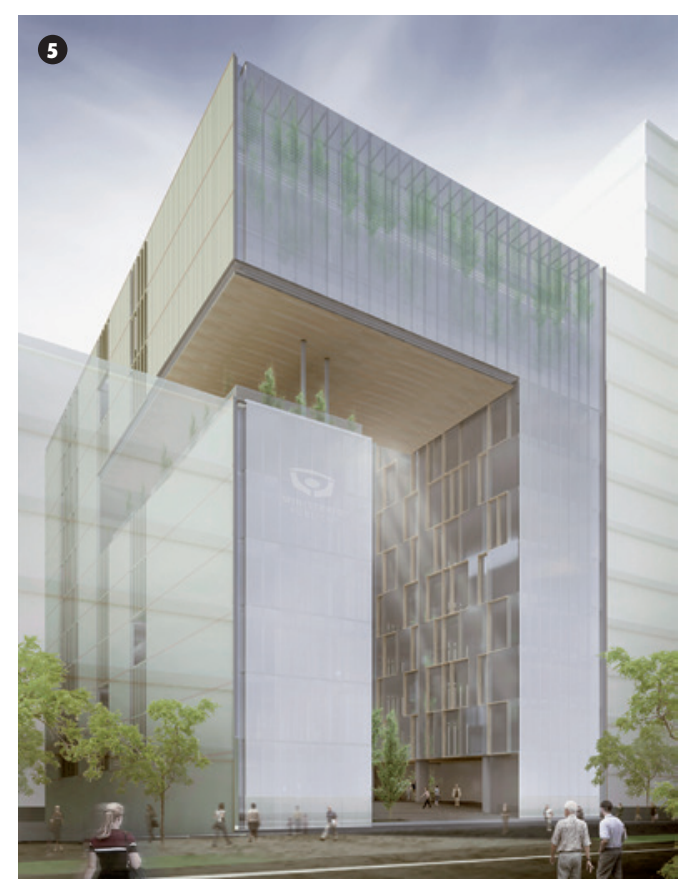

En el último tiempo hemos venido a observar la emergencia de una nueva generación de arquitectos interesados en acceder al diseño de arquitectura pública pese a todas las limitaciones y trabas que el sistema presenta. Solo como referencia, convendría citar el caso de la oficina Lateral, que junto al arquitecto Luis Corvalán, han resultado ganadores de la licitación para el Edificio de la Fiscalía Nacional y a la oficina HLPS, que junto con ganar el Concurso para el Parque Cultural de Valparaíso ya en pleno funcionamiento, más recientemente se han adjudicado el proyecto para el Teatro Regional de La Serena. Sin embargo, para que este interés se condense, es imprescindible considerar un avance hacia el establecimiento de un sistema de concursos y acceso al diseño de espacios y obras públicas masivo, inclusivo y transparente. De esta forma, podremos contribuir a solidificar el espacio del bien común y a reforzar lo que se ha venido en denominar como capacidades urbanas, "porque esas capacidades son propiedades sistémicas dirigidas a asegurar la ciudadanía, es decir, un espacio complejo que prospera con la diversidad y tiende a clasificar el conflicto en un civismo fortalecido" (Sassen, 2014: 29). 
6. Licitación Teatro Regional de La Serena. HLPS Arquitectos. Fuente: www.plataformaarquitectura.cl

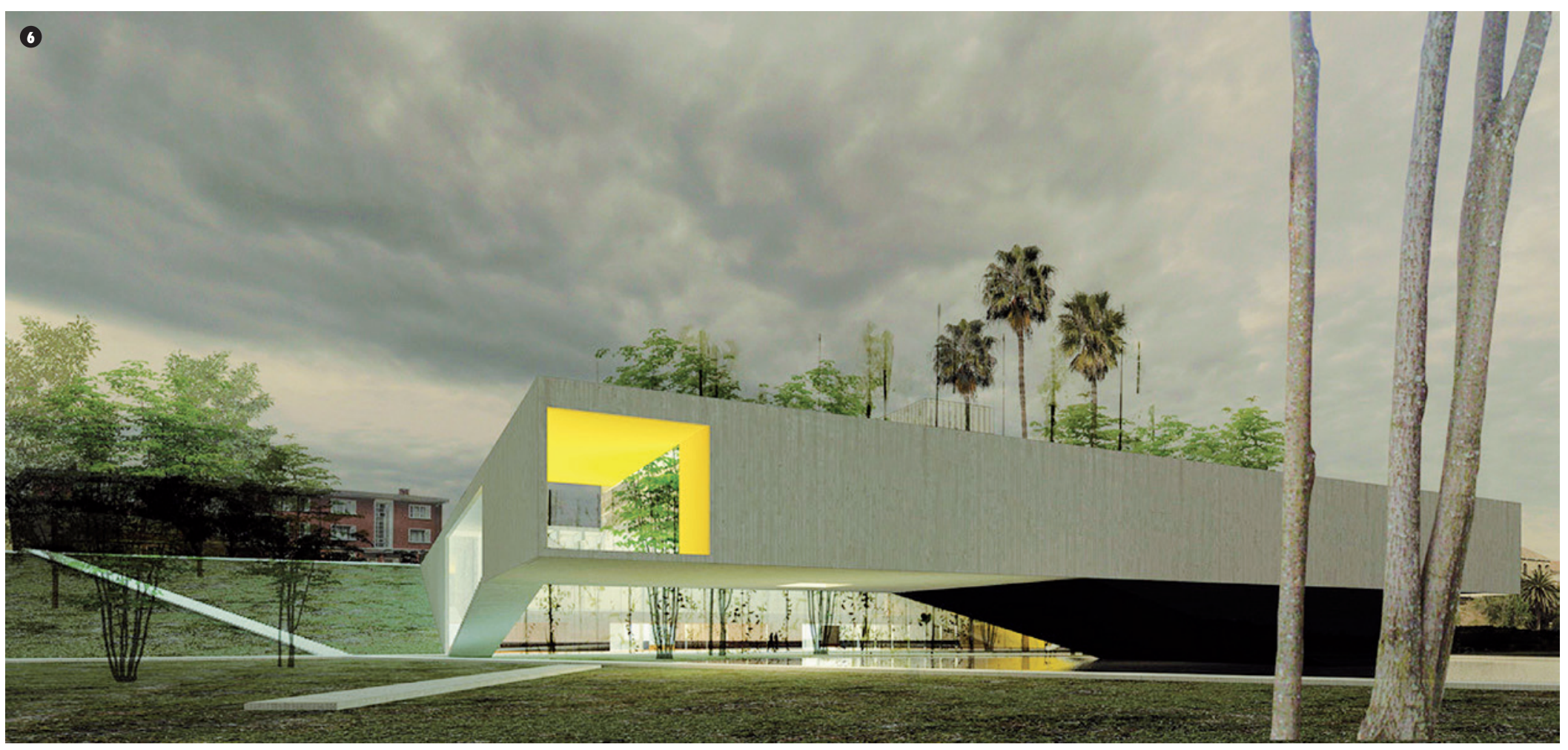

El cambio entonces debe estar orientado hacia una política de promoción de la arquitectura pública que modifique los procesos de selección y evaluación y que ponga el acento en la calidad de las propuestas presentadas. Considerando la cualidad transformadora de la arquitectura para sus habitantes y para las condiciones de mejora de la vida urbana, las optimizaciones que puedan emprenderse sobre el sistema de acceso al diseño de la arquitectura pública podrá saldar la deuda de la disciplina con la ciudad y volver entonces a conciliar el ejercicio de nuestra profesión con la ciudadanía.

\section{BIBLIOGRAFÍA}

Adria, M. (201 1). "Postales Chilenas". En: Blanca Montaña. Arquitectura reciente en Chile. Santiago de Chile, Ediciones Puro Chile.

Mardones, P. (2008). "Chile: genio y figura". En: Revista 2G Dossier. Iberoamérica. Madrid, Editorial Gustavo Gili.

Matus, C.; Atria, M.; Torrent, H. (2012). Estudio del sistema de licitaciones públicas para e fortalecimiento de los concursos de arquitectura en Chile. Santiago de Chile, Consejo Nacional de la Cultura y las Artes. Web www.cultura.gob. $\mathrm{cl}$ /estudios/observatorio-cultural.

Matute, P. (2010). "La ciudad concursada". En: Intenciones de ciudad. Arquitectura, cultura y territorio. Santiago de Chile, Fondo Nacional de la Cultura y las Artes.
Molina, C. (2014). Concursos de Arquitectura en Chile. Su aporte al desarrollo cultural y a la calidad de vida. Santiago de Chile, Consejo Nacional de la Cultura y las Artes.

Montealegre B., A. (2007). "Derechos, fines y medios. La declinación del concurso de arquitectura". En: Revista ARQ N 67, Concursos de Arquitectura. Santiago de Chile, ARQ Ediciones.

Palma, C. (2006). "Truly, Madly, Deeply". En: Revista $A R Q N^{\circ} 64$, Chile: dentro y fuera. Santiago de Chile, ARQ Ediciones.

Pérez, F. (2007). "Tras los concursos". En: Revista ARQ $N^{\circ}$ 67, Concursos de Arquitectura. Santiago de Chile, ARQ Ediciones.

Piga, J. (2008). Cuatro concursos de arquitectura pública. Santiago de Chile, Dirección de Arquitectura, Ministerio de Obras Públicas.

Sassen, S. (2014). "¿Hablan las ciudades?". En: Habla ciudad. Ciudad de México, Ediciones Arquine. 